\title{
Transaortic repair for the moderate functional mitral regurgitation
}

\author{
Nan Ma, MD, PhD, Hao Liu, MD, PhD, Fangbao Ding, MD, PhD, and Ju Mei, MD, PhD, Shanghai, China
}

As many as $75 \%$ of patients undergoing aortic valve replacement also have functional mitral regurgitation (FMR). How to deal with concomitant moderate FMR remains controversial. ${ }^{1,2}$ Repair of moderate mitral regurgitation with a prosthetic ring may lead to physiologic annular dynamic inhibition and transmitral flow influence, costing more in operative time and medical expenses. If left alone, however, moderate FMR will affect cardiac function and delay surgical rehabilitation. Residual regurgitation may deteriorate, necessitating reoperation. ${ }^{3}$ Fortunately, these problems can be solved by our technique when the concomitant moderate FMR is repaired through a transaortic approach.

\section{CLINICAL SUMMARY}

A total of 25 patients undergoing aortic valve or aortic root procedures underwent transaortic mitral valve repair from January 2006 to June 2011. There were 18 male $(72 \%)$ and 7 female $(28 \%)$ patients, with a mean age of $57.9 \pm 9.6$ years. All those patients could be subclassified as having type I mitral regurgitation according to the Carpentier classification. The causes of aortic valve disease can be classified as follows: degenerative (14 patients), rheumatic (4 patients), 2-leaflet abnormity ( 2 patients), and Marfan syndrome (5 patients). The predominant aortic valve diseases were aortic regurgitation in 18 patients, aortic stenosis 13 in patients, and combined aortic regurgitation and aortic stenosis in 4 patients.

Intraoperative transesophageal echocardiography (TEE) confirmed the previous findings. Conventional cardiopulmonary bypass was established through a median sternotomy, and peripheral cardiopulmonary bypass through the femoral artery was established through a transverse parasternal incision over the third intercostal space. The mitral valve was approached through the incision of the anterior wall of the ascending aorta or aortic root. Leaflet, annulus, chordae, and papillary muscles were exposed after resection of the aortic valve. There was no organic disease of the mitral valve. We used 2-0

\footnotetext{
From the Department of Cardiothoracic Surgery, Xinhua Hospital, School of Medicine, Shanghai Jiaotong University, Shanghai, China.

Disclosures: Authors have nothing to disclose with regard to commercial support.

Received for publication June 7, 2013; revisions received Aug 19, 2013; accepted for publication Aug 29, 2013.

Address for reprints: Ju Mei, MD, PhD, Department of Cardiothoracic Surgery, Xinhua Hospital, School of Medicine, Shanghai Jiao Tong University, 1665 Kong Jiang Rd, Shanghai (200092), P. R. China (E-mail: ju_mei@yahoo.cn). J Thorac Cardiovasc Surg 2014;147:526-7

$0022-5223 / \$ 36.00$

Copyright (C) 2014 by The American Association for Thoracic Surgery http://dx.doi.org/10.1016/j.jtcvs.2013.08.081
}

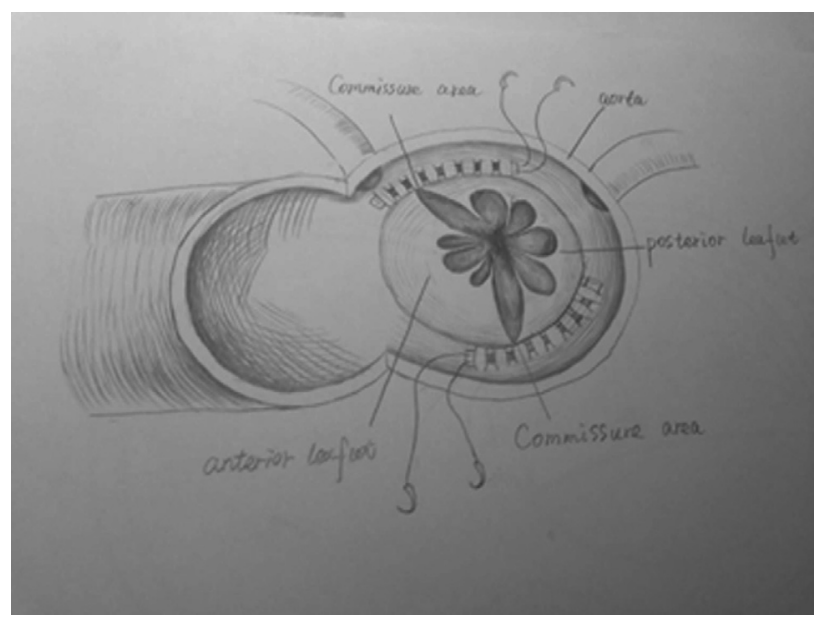

FIGURE 1. Suture with 2 pledgets from the posterior annulus to the anterior annulus at the 2 commissural areas through a transaortic approach.

polypropylene (Prolene; Ethicon Endo-Surgery, Inc, Somerville, NJ) mattress suture with 2 pledgets from posterior annulus to anterior annulus at the 2 commissural areas (Figure 1). This technique took small anchoring suture bites of the anterior annulus and progressively took larger bites along the adjacent posterior annulus, then the length of the posterior annulus significantly decreased and the posterior annulus came close to the anterior annulus (Figure 2). In all, 16 aortic valve replacement procedures, 8 Bentall procedures, and 1 Wheat procedure were then performed. Concomitant procedures included 2 bypass procedures, 1 ligation of persistent ductus arteriosus, 4 maze IV procedures, and 5 tricuspid valve repair procedures. TEE was carried out before the end of each procedure.

Lessened mild residual regurgitation was found in 2 patients through TEE. No mitral valve regurgitation or stenosis was found in the other 23 patients. Operative times for cardiopulmonary bypass and aortic crossclamp were $75.9 \pm 20.4$ minutes and $56.5 \pm 16.4$ minutes, respectively. No deaths occurred in this population. The left ventricular diastolic diameter decreased from $57.8 \pm 6.7 \mathrm{~mm}$ to 52.8 $\pm 6.5 \mathrm{~mm}$. The ejection fraction was $46.8 \% \pm 5.0 \%$. There was no significant variation in diameter of left atrium, mitral valve orifice area, mean gradient pressure, or peak gradient pressure between the left atrium and left ventricle. The results before discharge of mitral valve repair, according to TEE and transthoracic echocardiography, were the same.

All patients were followed up for a mean interval of $50.4 \pm 25.3$ months. There were 3 patients who had recurrence or aggravation of regurgitation. One had mild residual regurgitation, which developed to moderate 


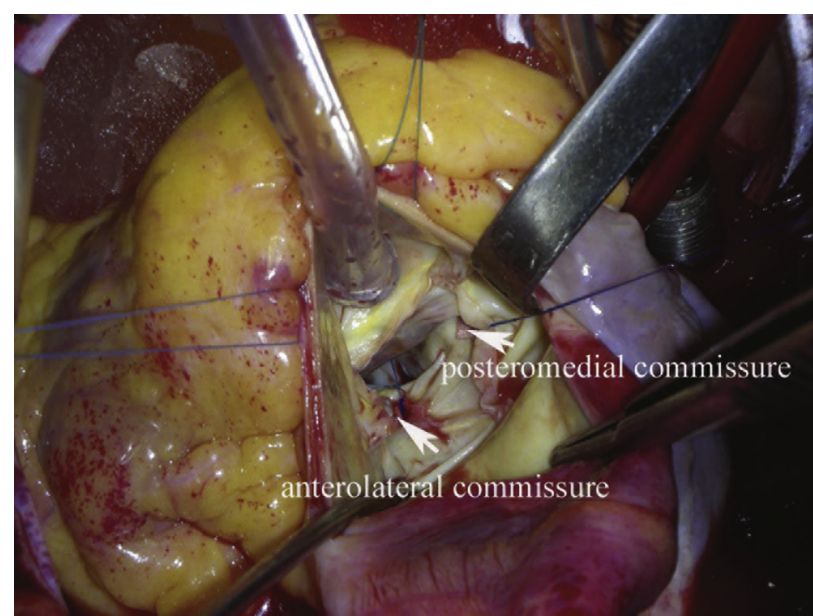

FIGURE 2. Suture annuloplasty through a transaortic approach.

regurgitation at the ninth month after the procedure. The other 2 patients had recurrence of mild regurgitation at the third and 30th months. Freedom from mitral regurgitation was $86.9 \%$.

\section{DISCUSSION}

Suture annuloplasty can maintain the physiologic motion and spatial structure of the mitral annulus without prosthetic ring. Commissural suture, a type of suture annuloplasty, can effectively recover the mitral valve function by decreasing the length of the posterior annulus. ${ }^{4}$ Our data showed good results of the commissural suture for moderate FMR. We could get excellent mitral valve exposure through the transaortic approach when the diameter of aortic annulus was enlarged. A retractor was used to withdraw the chordae to reveal the commissural area and avoid entanglement. Commissural suture performed through the transaortic approach was just as easy as through a routine approach. Nevertheless, the technique requires experience when executing it on an aortic annulus of normal or small size diameter. Transaortic repair was suitable, especially when upper median sternotomy or transverse parasternal incision over the third intercostal space was used. The advantages included no bleeding from an atrial incision and no extensive release of adhesions in reoperations. ${ }^{5}$ It is thus safe, effective, and convenient to repair moderate FMR by commissural suture through transaortic approach when the procedure of aortic valve or aortic root replacement is performed.

We thank Drs Saie Shen, Yuan Sun, and Yin Cai and Mss Huihua Chen and $\mathrm{Yu} \mathrm{Su}$ for their excellent clinical support and follow-up data collection. We thank Dr Ting Zhang for the language revision. We thank Mr Dai Yang for the diagram.

\section{References}

1. Enriquez-Sarano M, Akins CW, Vahanian A. Mitral regurgitation. Lancet. 2009; 373:1382-94

2. Wan CK, Suri RM, Li Z, Orszulak TA, Daly RC, Schaff HV, et al. Management of moderate functional mitral regurgitation at the time of aortic valve replacement: is concomitant mitral valve repair necessary? J Thorac Cardiovasc Surg. 2009;137: 635-40.

3. Suri RM, Aviernos JF, Dearani JA, Mahoney DW, Michelena HI, Schaff HV, et al Management of less-than-severe mitral regurgitation: should guidelines recommend earlier surgical intervention? Eur J Cardiothorac Surg. 2011;40:496-502.

4. Fundarò P, Tartara PM, Villa E, Fratto P, Campisi S, Vitali EO. Mitral valve repair: is there still a place for suture annuloplasty? Asian Cardiovasc Thorac Ann. 2007; $15: 351-8$

5. Koizumi J, Nakajima T, Izumoto H, Ohsawa S, Ishihara K, Kawazoe K Transaortic mitral valve repair combined with a modified Bentall procedure in a patient with Marfan's syndrome: report of a case. Surg Today. 2004;34:774-6.

\title{
An alternative technique for surgical repair of pulmonary dissection
}

\author{
Gianfranco Montesi, MD, ${ }^{\mathrm{a}}$ Sabina Caciolli, MD, ${ }^{\mathrm{b}}$ Manlio Acquafresca, MD, ${ }^{\mathrm{b}}$ and \\ Sandro Gelsomino, $\mathrm{MD}, \mathrm{PhD},{ }^{\mathrm{a}}$ Florence, Italy
}

Pulmonary artery dissection (PAD) is a rare but lifethreatening event that usually occurs with other congenital

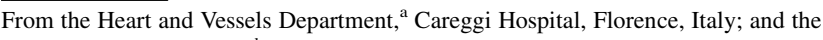
Radiology Department, ${ }^{\mathrm{b}}$ Careggi Hospital, Florence, Italy.

Disclosures: Authors have nothing to disclose with regard to commercial support.

Received for publication May 9, 2013; revisions received July 30, 2013; accepted for publication Sept 6, 2013; available ahead of print Oct 30, 2013.

Address for reprints: Sandro Gelsomino, MD, PhD, Department of Heart and Vessels,

Careggi Hospital, Largo Brambilla 3, 50134, Florence, Italy (E-mail: sandro.

gelsomino@libero.it).

J Thorac Cardiovasc Surg 2014;147:527-9

$0022-5223 / \$ 36.00$

Copyright (c) 2014 by The American Association for Thoracic Surgery

http://dx.doi.org/10.1016/j.jtcvs.2013.09.019
}

cardiac defects or as a complication of chronic pulmonary arterial hypertension. ${ }^{1}$ Other causes of PAD include connective tissue disorders, right heart endocarditis, amyloidosis, trauma, and severe atherosclerosis. Only few cases of PAD treated surgically have been reported in the literature, ${ }^{2-4}$ and no definitive approach has been established. We describe an alternative technique for surgical repair of PAD.

\section{CLINICAL SUMMARY}

A 46-year-old patient with Ehlers-Danlos syndrome who had undergone Bentall composite aortic root replacement for type A aortic dissection 10 years previously was 